Wiefel, Andreas; Titze, Karl; Kuntze, Lena; Winter, Mirja; Seither, Corinna; Witte, Ben; Lenz, Klaus; Grüters, Annette; Lehmkuhl, Ulrike

\title{
Diagnostik und Klassifikation von Verhaltensauffälligkeiten bei Säuglingen und Kleinkindern von 0-5 Jahren
}

Praxis der Kinderpsychologie und Kinderpsychiatrie 56 (2007) 1, S. 59-81

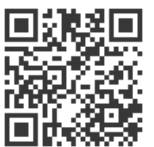

Quellenangabe/ Reference:

Wiefel, Andreas; Titze, Karl; Kuntze, Lena; Winter, Mirja; Seither, Corinna; Witte, Ben; Lenz, Klaus; Grüters, Annette; Lehmkuhl, Ulrike: Diagnostik und Klassifikation von Verhaltensauffälligkeiten bei Säuglingen und Kleinkindern von 0-5 Jahren - In: Praxis der Kinderpsychologie und Kinderpsychiatrie 56 (2007) 1, S. 59-81 - URN: urn:nbn:de:0111-opus-30450 - DOI: 10.25656/01:3045

\section{Vandenhoeck \& Ruprecht}

\section{VGR}

http://www.v-r.de

\section{Nutzungsbedingungen}

Gewährt wird ein nicht exklusives, nicht übertragbares, persönliches und beschränktes Recht auf Nutzung dieses Dokuments. Dieses Dokument ist ausschließlich für den persönlichen, nicht-kommerziellen Gebrauch bestimmt. Die Nutzung stellt keine Übertragung des Eigentumsrechts an diesem Dokument dar und gilt vorbehaltlich der folgenden Einschränkungen Auf sämtlichen Kopien dieses Dokuments müssen alle Urheberrechtshinweise und sonstigen Hinweise auf gesetzlichen Schutz beibehalten werden. Sie dürfen dieses Dokument nicht in irgendeiner Weise abändern, noch dürfen Sie dieses Dokument für öffentliche oder kommerzielle Zwecke vervielfältigen, öffentlich ausstellen, aufführen, vertreiben oder anderweitig nutzen.

Mit der Verwendung dieses Dokuments erkennen Sie die Nutzungsbedingungen an.

\section{Terms of use}

We grant a non-exclusive, non-transferable, individual and limited right to using this document.

This document is solely intended for your personal, non-commercial use. Use of this document does not include any transfer of property rights and it is conditional to the following limitations: All of the copies of this documents must retain all copyright information and other information regarding legal protection. You are not allowed to alter this document in any way, to copy it for public or commercial purposes, to exhibit the document in public, to perform, distribute or otherwise use the document in public.

By using this particular document, you accept the above-stated conditions of use.

\section{Kontakt / Contact:}

peDOcs

DIPF | Leibniz-Institut für Bildungsforschung und Bildungsinformation

Informationszentrum (IZ) Bildung

E-Mail: pedocs@dipf.de

Internet: www.pedocs.de

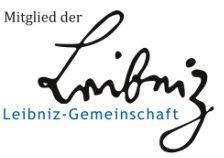




\section{Diagnostik und Klassifikation von Verhaltensauffälligkeiten bei Säuglingen und Kleinkindern von 0-5 Jahren}

Andreas Wiefel, Karl Titze, Lena Kuntze, Mirja Winter, Corinna Seither, Ben Witte, Klaus Lenz, Annette Grüters und Ulrike Lehmkuhl

\section{Summary \\ Diagnostic Classification of Mental Disorders in Infants and Toddlers Age 0 to 5}

The Article is working on developmental psychopathology, diagnosis, and treatment of behavioral problems in infants and toddlers age 0 to 5 . An overview of the literature about the international discussion is given. In particular diagnostic classification is elaborated by mentioning the revision of „Diagnostic Classification 0-3 (DC: 0-3R)“ and „Research Diagnostic Criteria-Preschool Age (RDC-PA)“. State of the art and clinical implication is reported on the basis of principal considerations on infant psychiatry. The american practice parameters become adapted and a working title for diagnostic formulation is given. More research should be done against the background of the introduced standards.

Prax. Kinderpsychol. Kinderpsychiat. 56/2007, 59-81

\section{Keywords}

Diagnostic Classification 0-3 (DC: 0-3R) - Infant Psychiatry - Parent-Infant-InteractionPractise Parameters - Research Diagnostic Criteria Preschool Age (RDC-PA)

\section{Zusammenfassung}

Der Artikel gibt einen zusammenfassenden Überblick über die internationale Diskussion und den Stand der Literatur zu Grundsatzproblemen der Psychopathologie, diagnostischen Problemen und Möglichkeiten sowie zu Behandlungsstandards bei Verhaltensauffälligkeiten in der Altersklasse von der Geburt bis zum Vorschulalter (0 bis 5 Jahre). Es wird auf die aktuelle Revision der „Diagnostic Classification 0-3 (DC: 0-3R)“ der Arbeitsgruppe Zero To Three (ZTT) im August 2005 und die Formulierung von Forschungskriterien, „Research Diagnostic Criteria-Preschool Age (RDC-PA)“ eingegangen. Der Stand der Literatur wird referiert und klinische Implikationen werden aufgezeigt. Grundsätzliche Fragestellungen einer frühkindlichen Psychopathologie werden besprochen und geeignete Untersuchungsverfahren vorgestellt. Es wird ein Arbeitsvorschlag für ein Standardvorgehen im Bereich diagnostischer Zuordnung bei Verhaltensauffälligkeiten bei Kindern von 0 bis 5 Jahren gegeben. Auf der Grundlage der vorgeschlagenen Standards sollten weitere Untersuchungen zur Evidenzbasierung der vorgestellten diagnostischen und therapeutischen Maßnahmen bei Säuglingen und Kleinkindern mit Verhaltensauffälligkeiten durchgeführt werden. 


\section{Schlagwörter}

Diagnostische Klassifikation (DC: 0-3R) - Säuglings- und Kleinkindpsychiatrie - Eltern-Kleinkind-Interaktion - Leitlinienbehandlung - Forschungskriterien im Vorschulalter (RDC-PA)

\section{Grundsatzprobleme der Psychopathologie des Kleinkindalters}

Im englischen Sprachgebrauch stehen ,infant psychiatry“ und „infant mental health“ traditionell nahe beieinander und sorgen doch für anhaltende Diskussionen um Gemeinsamkeiten und Abgrenzung zwischen Salutogenese und Psychopathologie (Zeanah 1997a,b). Im Deutschen wirken die Begriffe „Säuglings- und Kleinkindpsychiatrie" und „Seelische Gesundheit in der frühen Kindheit" noch stärker antipodisch. Objektiv beinhalten beide klinische Entwicklungspsychologie und -psychopathologie sowie Diagnostik und Therapie von Verhaltensauffälligkeiten in der Altersklasse der Säuglinge und Vorschulkinder.

Im klinischen Rahmen sind dabei psychologische, pädiatrische und sozialpädiatrische sowie familientherapeutische, aber auch kinder- und jugendpsychiatrische und psychiatrische Aspekte zu berücksichtigen.

Bei der Definition des Altersspektrums zeigt sich eine erhebliche Interpretationsvielfalt. Die „Diagnostic Classification 0-3 (DC: 0-3)“ wurde rasch nicht nur bis zum dritten, sondern „durch das dritte Lebensjahr“ benutzt (ZTT 1994, 2005). Andere Autoren verstehen die Anwendbarkeit im Altersspektrum 0 bis 5 („,under five“) (Emde 2003, Guedeney et al. 2003). In der 2005 revidierten Version DC: 0-3R findet sich der Hinweis, dass die meisten Teilnehmer am Review-Prozess Kinder im Alter von 0-5 Jahren behandeln. Die „Research Diagnostic Criteria Preschool Age (RDC-PA)“ werden bis zum 7. Lebensjahr benutzt (Task force 2003, Scheeringa, pers. Mitteilung 2005).

Traditionell behandelten klinische Arbeiten zu psychopathologischen Auffälligkeiten dieser Altersgruppe vorrangig somatisch begründete Entwicklungsstörungen und den autistischen Formenkreis. Seit etwa zehn Jahren gibt es eine intensive Diskussion um das Wesen genuiner psychischer Störungen in der frühen Kindheit bei weniger kompakten Krankheitsentitäten. Die starke Abhängigkeit der emotionalen Entwicklung des Säuglings und Kleinkindes von seinem psychischen Umfeld lässt Gergely schon theoretisch zweifeln, ob eine intrinsische Störung unabhängig von einer gestörten Eltern-Kind-Beziehung existieren kann (Gergely 1996). Die Frage ist, ob es bei Säuglingen und Kleinkindern überhaupt intrapsychische Störungen, etwa im Sinne einer Neurose, geben kann (Bayley 1993, Carter et al. 2004, Papoušek et al. 2004, Thomas u. Clark 1998, Zeanah et al. 1997a).

Kleinkinder können schwerwiegende und anhaltende Verhaltensauffälligkeiten mit Beeinträchtigung der seelischen Entwicklung ausbilden (Laucht et al. 2000, Rutter u. Sroufe 2000). Fehlendes Problembewusstsein bei professionellen Gesundheitsdiensten führt dazu, dass Vorschulkinder trotz schwerwiegender Störungen im Sozialverhalten und der 
Kommunikation nur mit erheblichen Verzögerungen einer adäquaten Diagnostik zugewiesen werden (De Giacomo u. Fombonne 1998). „Wir sollten spätestens dann aufmerksam werden, wenn der Ablauf der alltäglichen Aktivitäten des Kindes durch psychische Phänomene behindert oder eingeschränkt ist“ (Emde 1999 nach Carter 2004, S. 110).

Die Rückübersetzung des englischen Begriffs „infant psychiatry“ verweist auf den lateinischen Wortstamm „infans“, was so viel bedeutet wie: „stumm, noch sehr klein“, eigentlich „nicht sprechend“. Bereits in der etymologischen Bedeutung findet sich der abgrenzende Hinweis auf die Zone der präverbalen Entwicklung. Damit ist zugleich die Hauptproblematik beschrieben, dass sich die kindzentrierte psychopathologische Diagnostik in dieser Altersgruppe auf die Beobachtung von non- und präverbalen Phänomenen beschränkt. Die wesentlichen psychiatrischen Methoden sprachlich und symbolgestützter Diagnostik und Psychotherapie basieren auf einem Reservoir für die Exploration, das hier nicht zur Verfügung steht. Selbst bei Kindern mit Sprachgebrauch jenseits des dritten Lebensjahrs sind durch noch ungenügend ausgeprägte oder nicht geförderte Symbolisierungsfähigkeit und „Theory of mind“ Einschränkungen zu berücksichtigen.

Durch die ausgeprägte Abhängigkeit von der primären Bezugsperson und dem psychosozialen Kontext ist die Untersuchung von Kleinkindern zusätzlich zu einem großen Anteil auf indirekte Äußerungen und Fremdberichte angewiesen. Ein wichtiger diagnostischer Fundus ist demnach zugleich eine Unschärfequelle, die nur durch Wissen und Erfahrung der Untersucher im Umgang mit bestehenden Diagnose- und Screening-Instrumenten kompensiert werden kann.

\subsection{Entwicklungstempo - Schnelle Wechsel in den Entwicklungsschritten}

Die Entwicklung in den ersten Lebensjahren erfolgt oft sprunghaft. Für die Einschätzung bestimmter Verhaltensweisen als pathologisch ist folglich eine gute Kenntnis und Orientierung an den normalen Entwicklungsschritten und deren Variationsbreite erforderlich. Wutanfälle sind beispielsweise für die Zeit um das zweite Lebensjahr aus entwicklungspsychologischen Gründen zur Autonomieentwicklung nicht nur physiologisch, sondern geradezu notwendig („terrible two“). Die psychopathologische Einschätzung erfolgt immer anhand der Beurteilung von Dauer, Frequenz, Intensität und Qualität des jeweiligen Verhaltens im alterstypischen Vergleich.

Ein anderes Beispiel: es macht einen Unterschied, ob ein Trauma vor oder nach dem 18. Lebensmonat erfahren wird, weil das Einsetzen der Symbolisierungsfähigkeit zu diesem Zeitpunkt zu unterschiedlichen Verarbeitungsmustern führt (Drell et al. 1993; Scheeringa et al. 1995).

\subsection{Kontextfaktoren}

Die meisten psychosozialen Risikofaktoren sind ihrer Natur und Wirkung nach variabel und nicht besonders spezifisch, aber sehr stabil (Sameroff 1998). 
Aggressives Verhalten mit zwei Jahren ist vorhersagbar aus dem Maß an mütterlicher Kompetenz und Wärme sowie ihrer Partnerzufriedenheit, wie sie schon lange vor Auftreten der Auffälligkeiten beim Kind existieren.

Die Symptombildung bei einer posttraumatischen Störung im frühen Kindesalter ist stärker von der Betroffenheit und der Copingreaktion eines Elternteils abhängig als von der Beteiligung oder Verletzung des betroffenen Kindes (Scheeringa et al. 1995).

„Der neue, bislang unbekannte Patient ist keine Person, sondern eine - allerdings asymmetrische - Beziehung" (Stern 1998).

Die Qualität der Beziehung zu der primären Bezugsperson ist der wichtigste Umgebungsfaktor. Er ist unter anderem determiniert von Einstellungen und Erfahrungen der Eltern, die schon lange vor der Geburt existieren.

Die Transmission innerer Repräsentationen von einer Generation zur nächsten ist hinlänglich bekannt und der Bindungsstil des Kindes in Abhängigkeit vom mütterlichen Bindungsmuster bereits in der Schwangerschaft vorhersagbar (Fonagy 2000, 2003).

Dennoch sind kindliche Bindungsmuster und Symptome sehr variabel in Abhängigkeit von der aktuell interagierenden primären Bezugsperson (Zeanah et al. 1997b, Fox 1991). Das macht die Abgrenzung genuiner emotionaler Störungen von passageren Entwicklungsproblemen schwierig.

Die englischen Komparative distress - disturbance - disorder verweisen schon lautmalerisch auf die cut-off-Problematik. Ein weiteres Problem ist die Abgrenzung und Einschätzung eines für das Kleinkind zunächst nur als Risiko vorhandenen Krankheitspotenzials, beispielsweise bei einer an sich noch nicht pathologischen unsicheren Bindung. Bisher wird diese Trennung zur Differenzierung einer Behandlungsindikation im Sinne der Richtlinien für Krankenkassenbehandlung (RVO, SGB V) strikt eingehalten (Resch 1996). Schließlich kann aufgrund der Pervasivität der Phänomene besonders im ersten Lebensjahr auch nur von einer geringen Spezifität einzelner Störungen ausgegangen werden.

Für die Praxis ist in diesem Bereich das subjektive Moment des (hier vor allem: elterlichen) „Leidensdrucks“ deshalb von ganz besonderer Bedeutung: Jenseits aller objektiven Kriterien kann z.B. schon moderates Schreien eines Säuglings bei gegebener Vulnerabilität eines Familiensystems zu Misshandlung führen, während die gleiche oder ausgeprägtere Symptomatik in einer anderen Familie problemlos verkraftet wird.

\subsection{Messprobleme}

Geeignete Messinstrumente zur Quantifizierung des Verhaltens sind die Voraussetzung, damit Kliniker und Forscher die Anzeichen und Symptome emotionaler Störungen in der frühen Kindheit beschreiben können (Keren et al. 2001).

Statt objektiver Messwerte finden wir jedoch große Unterschiede in den Berichten zwischen beiden Eltern oder zwischen Eltern und anderen Betreuern, ja sogar in der Einschätzung zwischen Untersucher und Eltern während einer gemeinsam beobach-

(C) Vandenhoeck \& Ruprecht GmbH \& Co. KG, Göttingen 
teten Episode. Eltern von Kindern, die wegen einer Verhaltensauffälligkeit in einer psychiatrischen Sprechstunde vorgestellt werden, berichten überwiegend externalisierende Symptome, während die Kinder selbst mehr internalisierende Symptome präsentieren (Hodges 1990, nach Zeanah 1997b). Kinder, die gar nicht explizit in einer Sprechstunde vorgestellt werden, zeigen im Gegensatz dazu mehr Symptome, als ihre Eltern wahrnehmen (Sawyer 1992, nach Zeanah 1997b). Andererseits berichten Eltern von Kindern mit Schlafstörungen mit größerer Validität über deren Schlafverhalten als Eltern von Kindern ohne Schlafprobleme (Minde 1993, nach Zeanah 1997b). Die elterlichen Erfahrungen im Vergleich mit anderen Kindern aus der Nachbarschaft oder Verwandtschaft beeinflussen die Berichte ebenso wie die Tatsache, ob von einem Einzel- oder Geschwisterkind die Rede ist (Stallard 1993, nach Zeanah 1997b).

Eltern neigen dazu, die Probleme kleiner Kinder als vorübergehend zu verstehen, und es besteht allgemein wenig Übereinstimmung in der Schweregradeinschätzung zwischen Eltern und Untersuchern (Pavuluri u. Luk 1996).

Wenn die primäre Informationsquelle ausschließlich bei den Eltern liegt, besteht immer die Gefahr, dass deren Projektionen und verzerrende Bindungsstile, die selbst Agens für gestörte Entwicklung sein können, unbewusst und verfälschend in die Bewertung des Kindes mit eingehen (Thomas u. Chess 1984).

Wegen dieser Einschränkungen ist es besonders wichtig, die verschiedenen zugänglichen Informationsquellen: Eltern, Fremdbeobachter und direkte Beobachtung des kindlichen Verhaltens in der Untersuchungssituation zu nutzen und vergleichend auszuwerten.

\section{Untersuchungsmethoden}

\subsection{Klinische Untersuchung (practise parameters)}

Praxisrichtlinien für ein diagnostisches Standardvorgehen sind in Anlehnung an die Richtlinien der American Academy of Child and Adolescent Psychiatry von 1995 vorgeschlagen worden (Thomas et al. 1997).

In diesen Richtlinien wird unterteilt in „infant“ für das Alter 0-12 Monate und „toddler“ für 12-36 Monate. Dies entspricht den deutschen Begriffen Säugling und Kleinkind, wobei der Übergang von Kleinkind zu Vorschulkind fließend ist und eine Gruppenbildung bis zum Ende des 4. Lebensjahr inhaltlich gerechtfertigt erscheint. Außerdem wird begrifflich geklärt, dass es neben den Eltern als Regelfall eine Reihe von abweichenden Konstellationen im sozial-familiären Gefüge gibt. Entscheidend ist, wer die „primäre Bezugsperson“ ist oder wer die „psychologischen Eltern“ sind. Das können Pflege- oder Adoptiveltern sein, Erzieher einer Einrichtung oder auch die Großeltern oder andere Personen aus der erweiterten Familie oder dem Freundeskreis. Im Untersuchungskontext muss diese zentrale Bindungsfigur des Kindes der Ansprechpartner sein.

(C) Vandenhoeck \& Ruprecht GmbH \& Co. KG, Göttingen 
Die Untersuchungsmethoden entsprechen im Grundsatz denen bei älteren Kindern und beinhalten zunächst Vorgeschichte und Familien- und Eigenanamnese. Abweichend ist eine immanente Ausrichtung auf Prävention und Risikoerkennung sowie eine viel intensivere Elternarbeit.

Bei der Erhebung des psychopathologischen Befundes schlagen die Richtlinien die Orientierung am „Infant and Toddler Mental Status Exam“ (ITMSE) vor (Tab. 1).

Tabelle 1: Psychopathologischer Befund im Kleinkindalter (in Anlehnung an ITMSE)

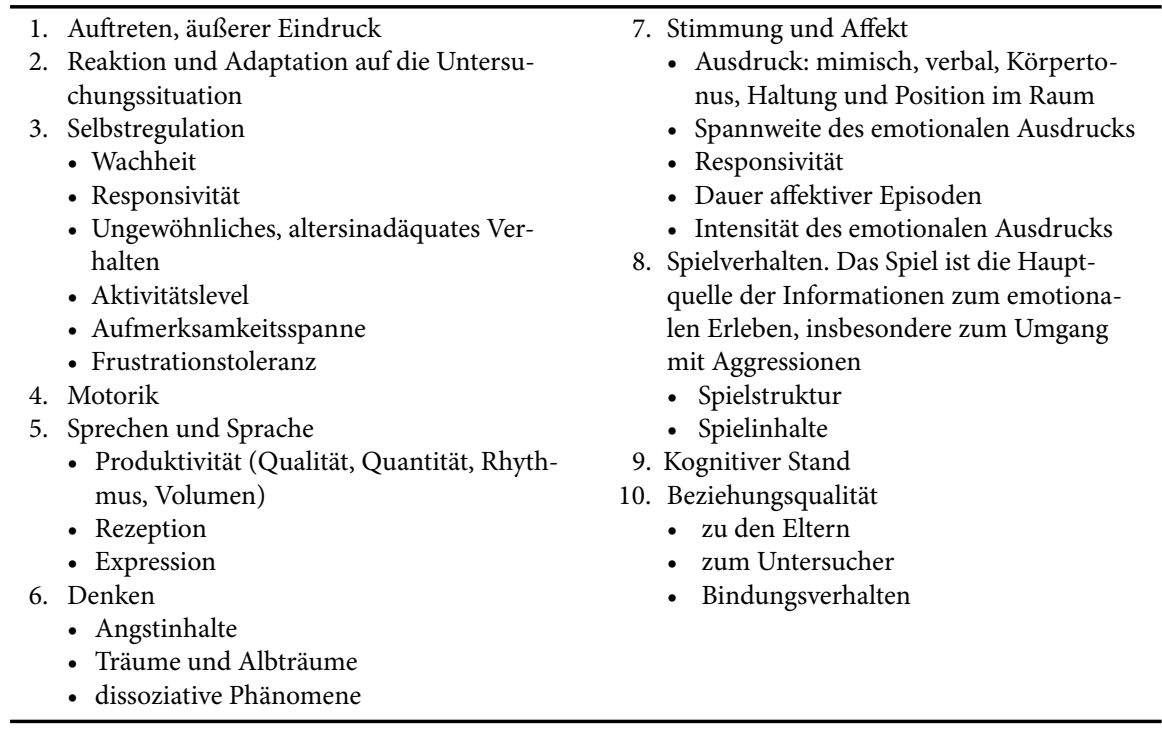

Hierbei gilt es aus unserer Erfahrung jedoch zu bedenken, dass es spätestens beim Versuch einer systematischen Dokumentation des psychopathologischen Befundes mit einem etablierten Instrument wie der „BADO“ erhebliche immanente Einschränkungen gibt. Bestimmte Phänomene wie „Denkstörungen“ oder „Sinnestäuschungen“ können aufgrund der oben genannten Limitationen weder erhoben noch verworfen werden und müssen somit schon aus systemlogischen Gründen als „nicht beurteilbar“ kodiert werden.

Wegen der raschen Entwicklungsschübe sind in der Regel mehrere Termine notwendig, und es sollte in verschiedenen Sozial- und Beziehungskontexten untersucht werden.

Ein multidimensionaler Ansatz ist obligat. Beteiligte Disziplinen sind: Geburtsmedizin und Pädiatrie mit Genetik, Ophthalmologie und Pädaudiologie sowie Entwicklungspsychologie, Logopädie und Ergotherapie.

(C) Vandenhoeck \& Ruprecht GmbH \& Co. KG, Göttingen 
Eine weitere Besonderheit ist die dyadische Sichtweise mit Schwerpunkt auf emotionalen Austauschprozessen. Zentraler diagnostischer Bestandteil ist deshalb die standardisierte Interaktionsbeobachtung. Die Richtlinien verweisen hier für genauere Ausführungen auf weitergehende Literatur.

Es wird unterstrichen, wie notwendig aber noch unvollständig die Entwicklung standardisierter Messinstrumente ist.

Es wird abschließend eine diagnostische Klassifikation mit den unten näher beschriebenen Klassifikationssystemen und eine ausführliche Therapieplanung gefordert.

\subsection{Dimensionale Messinstrumente}

Für viele Untersuchungsaspekte eignen sich dimensionale Verfahren besonders gut. Es steht eine Reihe entsprechender Instrumente zur Verfügung. Vorteilhaft ist ein Aufbau, der die Erstellung eines Profils aus Stärken und Schwächen erlaubt. Die gebräuchlichsten Verfahren seien hier kurz erwähnt.

\subsubsection{Symptomskalen}

Zur systematischen Erfassung einzelner Symptome stehen Fragebögen, Checklisten, Tests und strukturierte Interviews zur Verfügung.

\section{Fragebögen}

Für die weit verbreitete und gut geprüfte CBCL ist eine Variante für das Kleinkindalter entwickelt worden (Achenbach u. Rescorla 2000). Die CBCL 1,5 bis 5 besteht aus 99 Items. Sie misst emotionale und Verhaltensprobleme in den Bereichen internalisierende und externalisierende Probleme und liefert einen Gesamtproblemscore. Es gibt Unterskalen, die sich an DSM-Kriterien orientieren. Die Auswertung ergibt einen $\mathrm{t}$-Wert und es ist ein cut-off definiert. $\mathrm{t}$-Werte $<60$ werden als subklinisch, t-Werte $>63$ als klinisch auffällig gewertet. Das Instrument zeigt eine gute 8 -Tage Test-Retest Reliabilität. Die CBCL 1,5-5 unterscheidet gut zwischen einer klinischen und nicht-klinischen Population. Kinder, die an eine Fachambulanz verwiesen wurden, hatten in $74 \%$ auffällige Werte auf den Skalen internalisierende oder externalisierende Probleme. Kinder mit hohen CBCL Werten wurden 5-6 mal häufiger vorgestellt als Kinder mit niedrigen Werten.

\section{Verhaltensbeobachtung}

Die Vineland Skalen und das Home Observation for Measurement of the Environment (HOME)(Bradley 1985) sind die bekanntesten Instrumente zur Verhaltensbeobachtung.

(C) Vandenhoeck \& Ruprecht GmbH \& Co. KG, Göttingen 
66 A. Wiefel et al.

\section{Entwicklungstests}

Neben den international gebräuchlichen Bayley- und Griffith Scales sind im deutschen Sprachraum die Münchner funktionelle Entwicklungsdiagnostik und der ET 6-6 weit verbreitet.

Eine ausführliche Beschreibung weiterer Fragebögen, Instrumente zur Verhaltensbeobachtung und Entwicklungstests findet sich bei Carter (Carter et al. 2004) (Tab. 2).

\subsubsection{Interaktionsskalen}

Ein Kernstück der Diagnostik ist die Einschätzung der Interaktionsqualität zwischen dem Kind und seiner primären Bezugsperson. Auch dazu stehen theoriegeleitet verschiedene Instrumente zur Verfügung, und zwar sowohl behaviorale als auch qualitative, die meist am Konzept der Bindungstheorie orientiert sind (Papoušek 1995) (Tab. 3).

\subsection{Klassifikationssysteme}

Diagnostische Klassifikationssysteme dienen zur Definition und distinkten Abgrenzung verschiedener Krankheitsbilder sowie dem Austausch zwischen verschiedenen Behandlern einerseits und den Patienten und ihren Familien andererseits. Außerdem sind sie notwendige Voraussetzung für Therapieindikation und Outcomeforschung.

\subsubsection{ICD-10 und DSM-IV}

In beiden Klassifikationssystemen gilt das Grundprinzip der Unabhängigkeit von ätiologischen Annahmen.

Dieses Axiom wird mit jüngerem Alter immer problematischer, nimmt doch die Abhängigkeit vom Sozial- und Beziehungskontext proportional zu. Die Autoren der ICD-10 räumen schließlich ein, dass Interdependenzen nicht immer unberücksichtigt bleiben können (Remschmidt et al. 2001).

Im deutschen Sprachraum kommt die ICD-10 in einer Erweiterung als Multiaxiales Klassifikationssystem zur Anwendung, was für andere europäische und Nachbarregionen, z.B. Israel, nicht obligat ist und entsprechende Probleme birgt (Keren et al. 2003).

In den Leitlinien Sozialpädiatrie sind einige Vorschläge zur Verwendung von ICD-Diagnosen gemacht worden.

Für häufige Störungsbilder haben wir die Anwendbarkeit einzelner Kriterien aus der ICD-10 entsprechend ihrer Eignung für die frühe Kindheit aufgelistet (Tab. 4). 


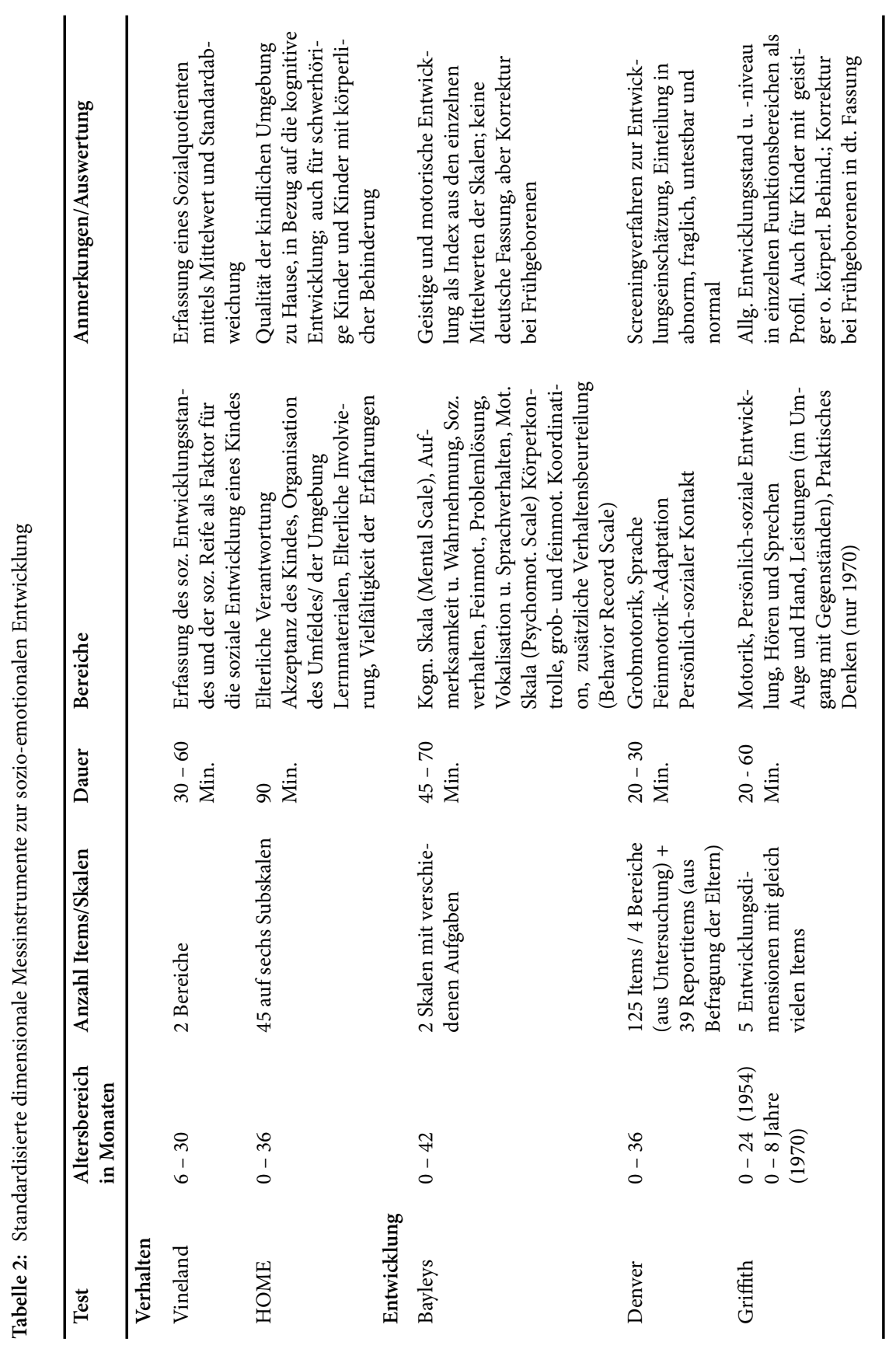

(C) Vandenhoeck \& Ruprecht GmbH \& Co. KG, Göttingen 
68 A. Wiefel et al.

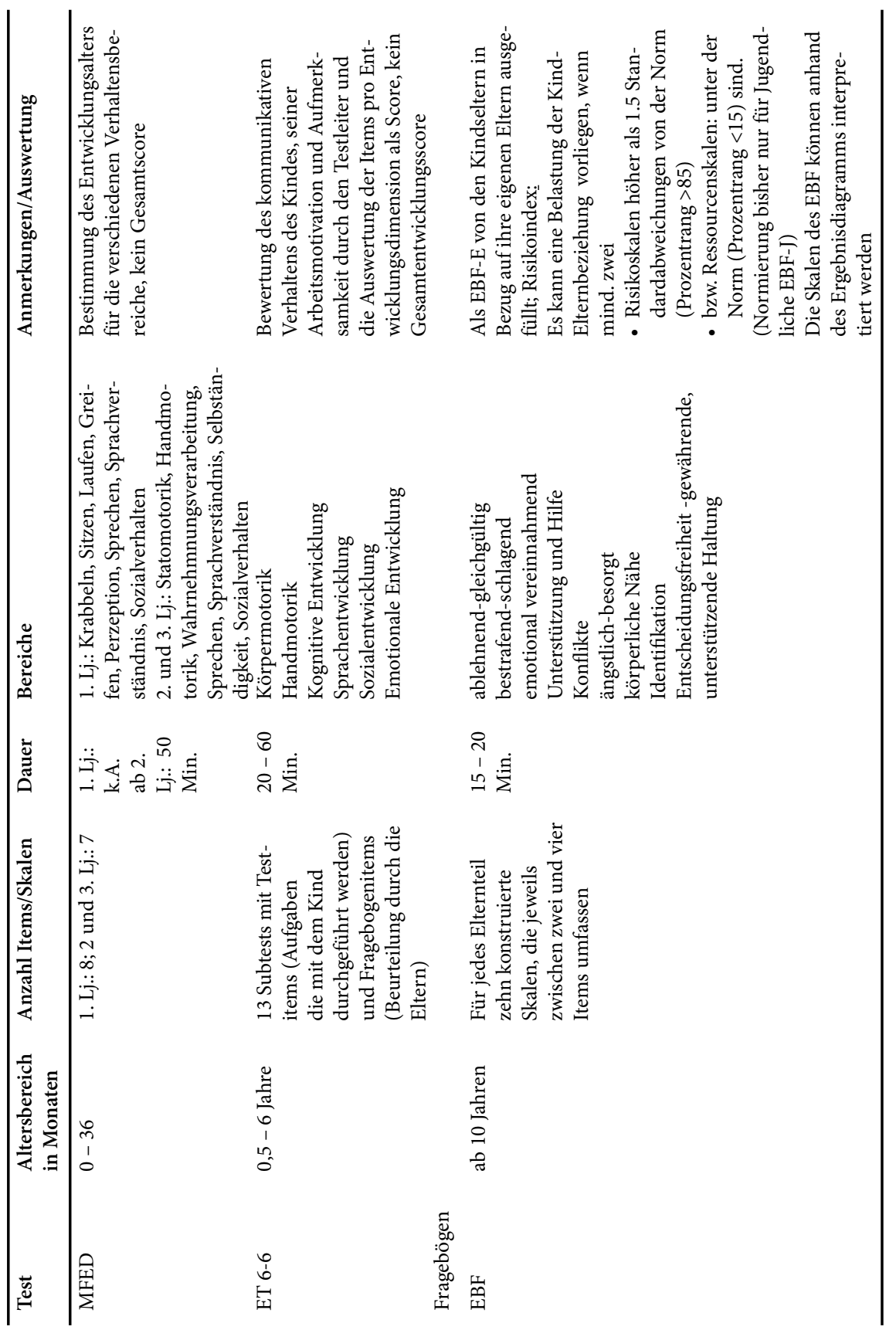

(c) Vandenhoeck \& Ruprecht GmbH \& Co. KG, Göttingen 


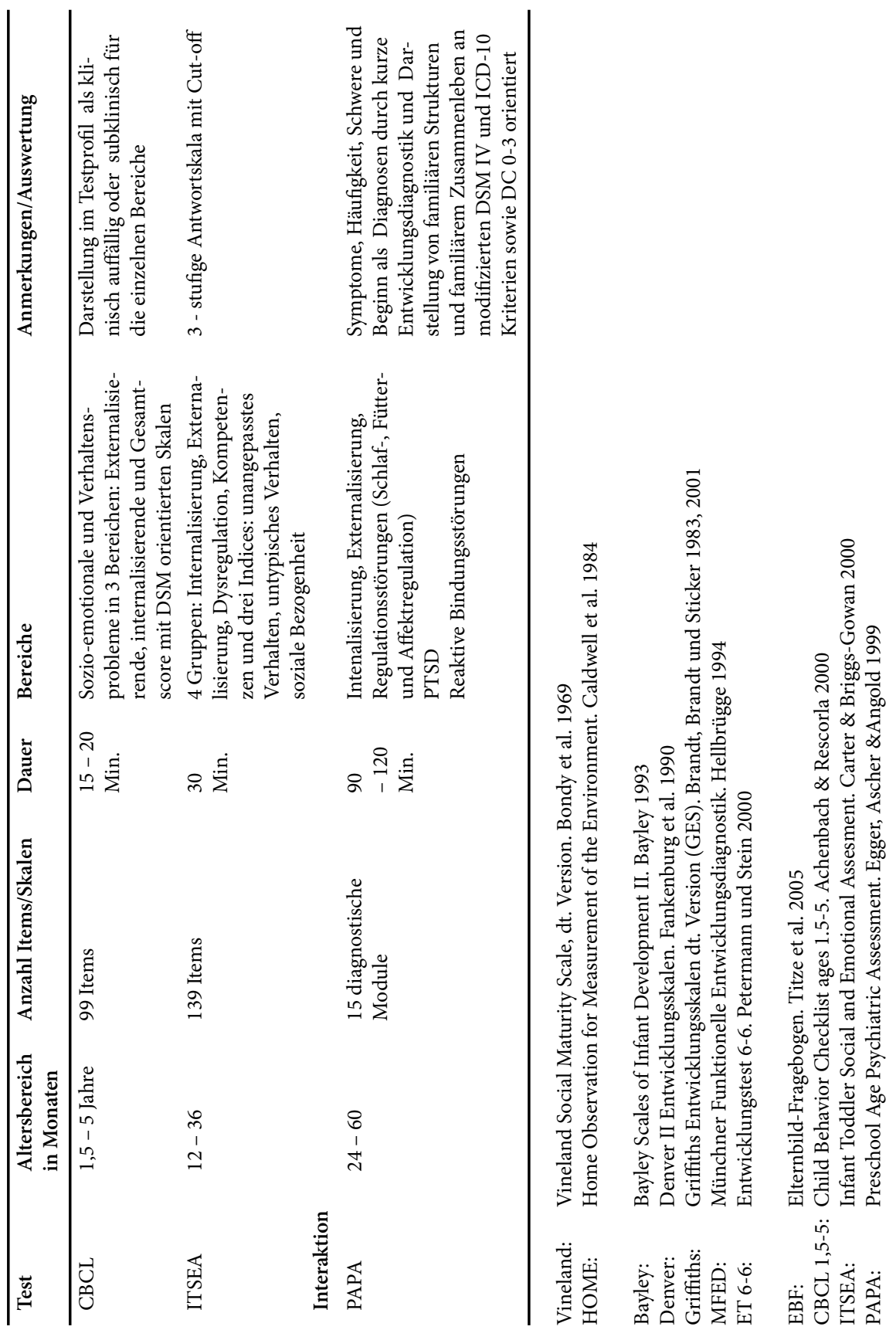

(C) Vandenhoeck \& Ruprecht GmbH \& Co. KG, Göttingen 
70 A. Wiefel et al.

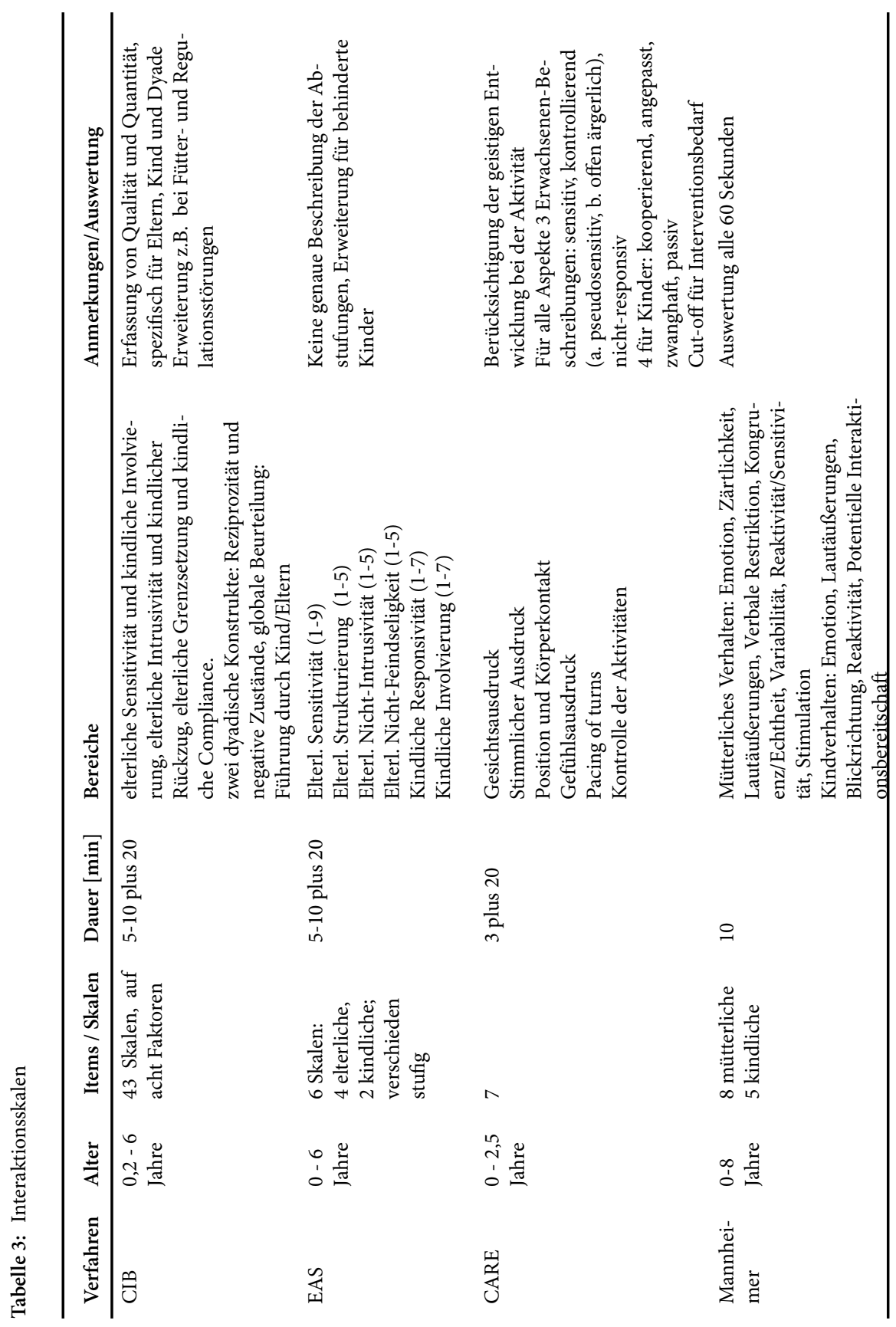

(c) Vandenhoeck \& Ruprecht GmbH \& Co. KG, Göttingen 


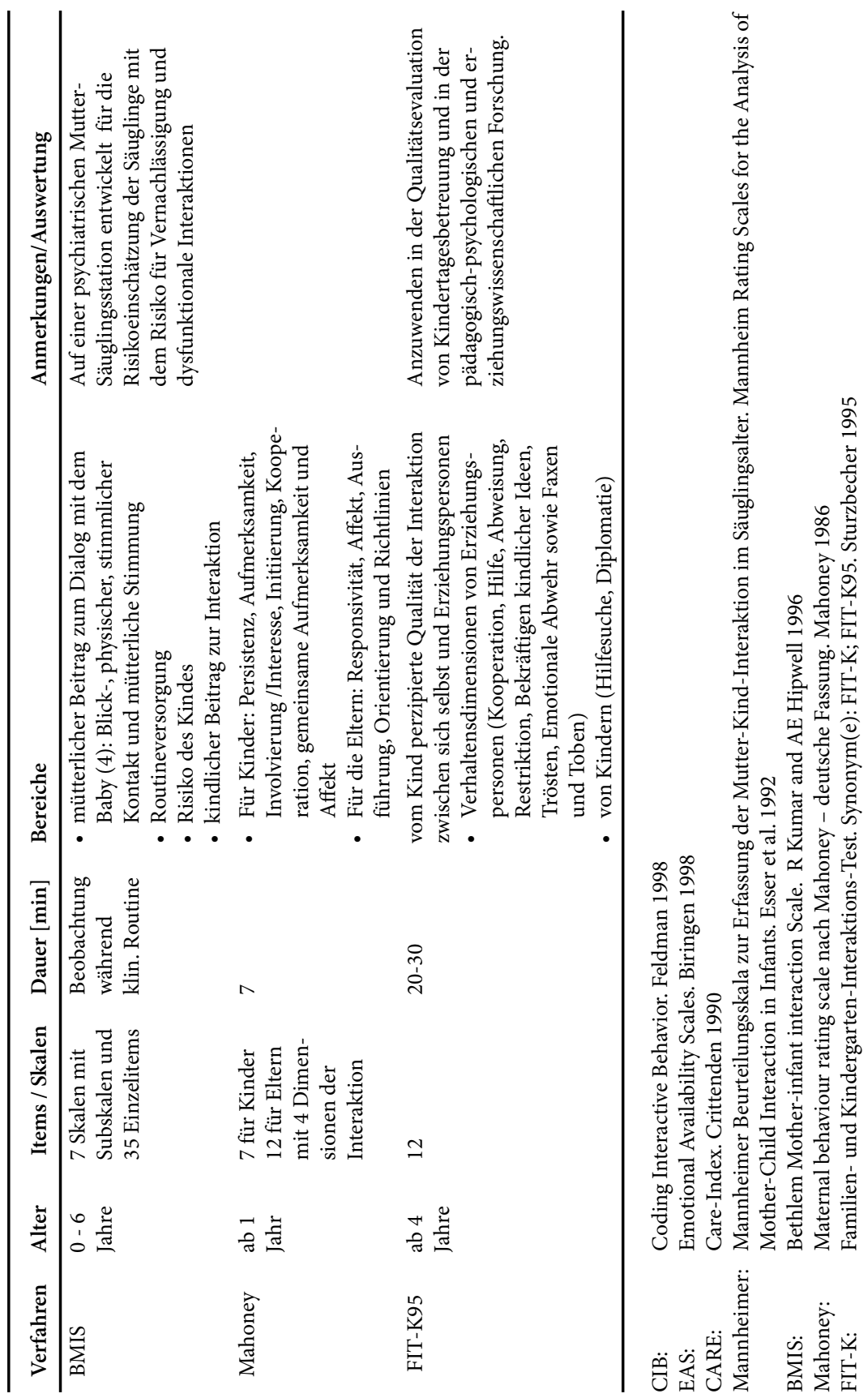

(C) Vandenhoeck \& Ruprecht GmbH \& Co. KG, Göttingen 
Tabelle 4: Eignung und Anwendbarkeit diagnostischer Kriterien der ICD-10 in der Altersgruppe der 0 bis 5-jährigen

\begin{tabular}{|c|c|c|}
\hline Code & Bezeichnung & Kommentar \\
\hline \multicolumn{3}{|c|}{ Gut geeignet } \\
\hline F84.x & Tiefgreifende Entwicklungsstörung & \\
\hline F93.3 & $\begin{array}{l}\text { Emotionale Störung mit Geschwis- } \\
\text { terrivalität }\end{array}$ & \\
\hline \multicolumn{3}{|c|}{ Eingeschränkt geeignet } \\
\hline F 43.x & Reaktionen auf Belastungen & unangemessene Symptom- und Zeitkriterien \\
\hline F93.x & emotionale Störungen & $\begin{array}{l}\text { eingeschränkt altersgerechte Kriterien, insbe- } \\
\text { sondere Trennungsangst/Ängste }\end{array}$ \\
\hline F94.x & Bindungsstörung & unangemessene Symptom- und Alterskriterien \\
\hline F98.2 & $\begin{array}{l}\text { Fütterstörung im frühen Kindes- } \\
\text { alter }\end{array}$ & $\begin{array}{l}\text { Gewichtsprobleme obligat, Ausschluss anderer } \\
\text { Verhaltensstörungen notwendig, keine Sub- } \\
\text { klassifikation möglich }\end{array}$ \\
\hline F91.x & Störungen des Sozialverhaltens & $\begin{array}{l}\text { keine altersgerechten Kriterien: Lügen, } \\
\text { Stehlen, Weglaufen bei Kleinkindern nicht } \\
\text { möglich. }\end{array}$ \\
\hline \multicolumn{3}{|c|}{ Nicht geeignet } \\
\hline F32.x & depressive Episoden & $\begin{array}{l}\text { keine altersgerechten Kriterien, unangemesse- } \\
\text { ne Zeitkriterien }\end{array}$ \\
\hline F51.x & nichtorganische Schlafstörungen & $\begin{array}{l}\text { keine altersgerechten Kriterien, z.B.: „fühlt } \\
\text { sich zur Schlafenszeit angespannt ... und emp- } \\
\text { findet ein Gedankenrasen“" }\end{array}$ \\
\hline F90.x & hyperkinetische Störungen & keine altersgerechten Kriterien \\
\hline
\end{tabular}

Explizit keine Berücksichtigung in der ICD-10 erhalten die alterstypischen Regulationsstörungen

\subsubsection{DC: $0-3 R$}

Die DC: 0-3R ist ein diagnostisches Instrument, das von der Arbeitsgruppe „Zero To Three“ (ZTT) am National Center for Infants, Toddlers and Families zur Berücksichtigung der Besonderheiten in der Altersklasse der 0 bis 3-jährigen eingeführt wurde (ZeroTo-Three 1994). Es soll die bestehenden Klassifikationssysteme DSM-IV und ICD-10 nicht ersetzen, sondern ergänzen. Es ist in seiner ersten Version inzwischen in mehrere Sprachen übersetzt und wird derzeit weltweit evaluiert (Dunitz et al. 1996; Frankel et al. 2004; Keren et al. 2001, 2003; Maestro et al. 2002; Maldonado-Durán et al. 2003; Wiefel et al. 2005). Eine erste Revision wurde im August 2005 herausgegeben.

\section{Anforderungen an Untersuchung und Diagnose mit der DC: 0-3R}

Alle für das Kind relevanten Lebensbereiche müssen mit den jeweils aktuellen diagnostischen Standards erfasst werden. Dazu zählen: aktuelle Symptome und Konstitutionsmerkmale, Erfassung von Entwicklungsstand und -geschichte, ausführliche Familienanamnese, anschließende Familien- und Elterndiagnostik und schließlich eine möglichst 
standardisierte und videodokumentierte Interaktionsdiagnostik. Eine vollständige Untersuchung erfordert in der Regel drei bis fünf Sitzungen von je 45 Minuten Dauer.

Die Achsen des Systems sind nur begrenzt mit denen von DSM-IV oder ICD-10 vergleichbar. Das junge Alter bewirkt die Ausrichtung an Entwicklungsparametern, die sich in der Beziehungsdynamik und den flexiblen Anpassungsleistungen widerspiegelt.

Auf der Achse I wird das psychiatrische Syndrom kodiert. In sieben Grundkategorien können Posttraumatische-, Affekt-, Anpassungs-, Regulations- Schlaf- und Essverhaltensstörungen sowie Störungen der Bezogenheit und der Kommunikation (autistischer Formenkreis) weiter differenziert werden. Achse II besteht aus der „Parent-Infant-Relationship Global-Assessment-Scale. (PIR-GAS) und erlaubt eine Einschätzung der Beziehungsqualität zu einer primären Bezugsperson auf einer Skala von 90 (gut adaptiert) bis 0 (misshandelnd) und die qualitative Beschreibung einer Beziehungsstörung auf einer Matrix von sechs Untergruppen: über/unterinvolviert, ängstlich/gespannt oder ärgerlich/feindselig.

Eine manifeste Beziehungsstörung wird unterhalb eines Scores von 40 diagnostiziert. Auf Achse III finden sich körperliche und medizinisch begründete Störungen. Mit der Achse IV werden die psychosozialen Stressfaktoren erfasst und die Achse V beschreibt das funktionell-emotionale Entwicklungsniveau (Tab. 5).

Das Manual gibt einige Grundsätze im diagnostischen Entscheidungsbaum vor:

- Wann immer vertretbar, soll bevorzugt eine Diagnose auf der Achse I vergeben werden. Wenn eine DSM-IV oder ICD-10-Diagnose besser passt, soll diese vergeben werden.

- Fachtherapeutische Diagnosen zu motorischer und sensorischer Entwicklung sowie von Sprache und Kommunikation werden auf der Achse III kodiert, ebenso alle medizinischen Diagnosen in Anlehnung an DSM-IV oder ICD-10.

- Bei eindeutigem Vorliegen eines Traumas soll eine posttraumatische Störung oder Anpassungsstörung vorrangig kodiert werden. Leichte Probleme von kurzer Dauer mit reaktivem Anteil gelten als Anpassungsstörung.

- Bei konstitutionell bedingten kindlichen Problemen mit der Folge maladaptiver Interaktionszirkel mit den Eltern soll eine Regulationsstörung vergeben werden.

- Wenn bei entsprechenden Symptomen diese ersten Regeln nicht greifen, soll eine affektive Störung diskutiert werden.

- Bei multiplen Problemen im kommunikativen und Sozialverhalten werden Multisystemische- und Bindungsstörung erörtert. Wenn die Probleme nur in bestimmten Situationen und nicht durchgängig auftreten, soll eine Anpassungs- oder Beziehungsstörung erwogen werden.

- Bei Vorliegen einer solitären Beziehungsstörung ohne Achse-I-Diagnose soll auch nur eine Achse-II-Diagnose vergeben werden.

- Reaktive Bindungsstörung soll nur vergeben werden, wenn eine inadäquate Versorgung im körperlichen, psychologischen oder emotionalem Bereich vorliegt, allerdings wird auch der psychoanalytische Begriff der „Frühstörung“ als ätiologisches Agens zitiert.

(c) Vandenhoeck \& Ruprecht GmbH \& Co. KG, Göttingen 
- Wenn neben Fütter- oder Schlafproblemen andere Symptome bestehen, dominieren letztere, wenn sie eine eigene Diagnose rechtfertigen. Dann soll diese vor Fütter- oder Schlafstörung vergeben werden.

- Bei Vorliegen mehrerer gestörter Bereiche soll der auffälligste kodiert werden, die Vergabe von zwei Diagnosen soll nur ausnahmsweise erfolgen.

Tabelle 5: Inhaltsverzeichnis/Kapitelüberschriften DC: 0-3R (Übersetzung Wiefel/Pfeiffer/Dunitz/Scheer)

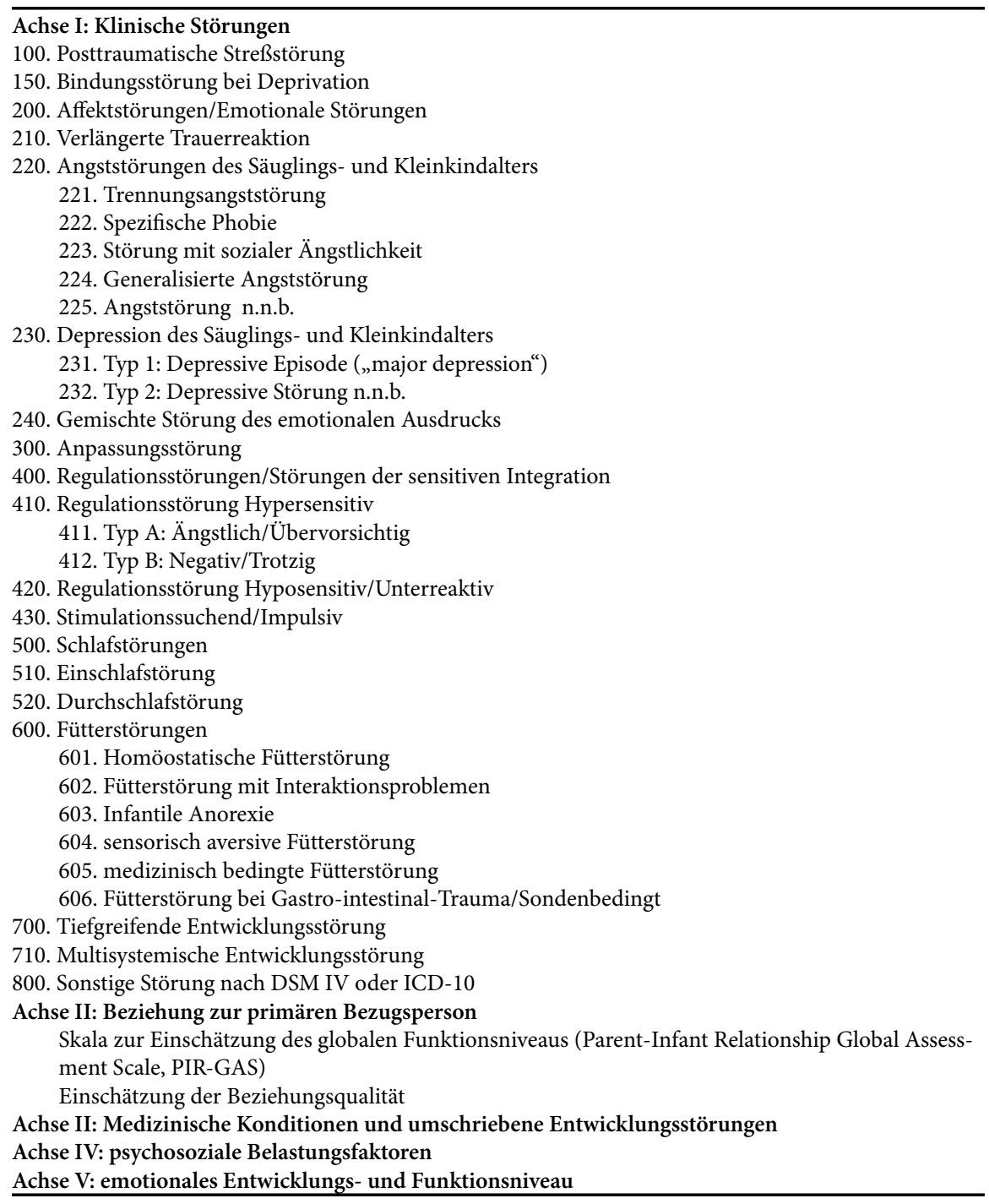

(C) Vandenhoeck \& Ruprecht GmbH \& Co. KG, Göttingen 


\section{Studien und Probleme mit der DC: 0-3, erste Version}

Erste Untersuchungen zu den Vorteilen und der Überlegenheit der DC: 0-3 gegenüber ICD-10 hat Dunitz-Scheer (1996) vorgenommen.

In einem special issue „DC: $0-3$ “ des Infant Mental Health Journals und in einer eigenen empirischen Arbeit sind von verschiedenen internationalen Arbeitsgruppen evaluative Vergleiche zwischen den Systemen vorgenommen worden (Guedeney et al. 2003; Cesari et al. 2003; Maldonado-Durán et al. 2003; Wiefel et al. 2005).

Dabei fanden sich durchgängig große Probleme mit der Applikation der Kriterien der klassischen Systeme, es wurden aber auch ungeklärte Fragen zur Anwendung der DC: 0-3 aufgeworfen (Tab. 6).

Tabelle 6: Studien zur DC: 0-3 aus dem Jahr 2003 (Infant Mental Health Journal, Special issue DC: 0-3) und 2005

\begin{tabular}{lllll}
\hline & $\begin{array}{l}\text { Guedeney } \\
\text { et al. (2003) }\end{array}$ & $\begin{array}{l}\text { Keren et } \\
\text { l. (2003) }\end{array}$ & $\begin{array}{l}\text { Maldonado } \\
\text { et al. (2003) }\end{array}$ & $\begin{array}{l}\text { Wiefel et al. } \\
(2005)\end{array}$ \\
\hline Fallzahl n= & 85 & 414 & 167 & 68 \\
Alter (Mittelwert in Monaten) & 18,3 & 19,0 & 21,2 & 23,0 \\
Geschlechterverhältnis m/w in \% & $53 / 47$ & $59 / 41$ & $59 / 41$ & $60 / 40$ \\
& & & & \\
Keine Diagnose & $23,5 \%$ & $55,2 \%$ & $9,5 \%$ & $8,8 \%$ \\
& & & & \\
Anpassungsstörungen & $8,2 \%$ & $8,2 \%$ & $23,2 \%$ & $5,9 \%$ \\
Affektstörungen & $20,0 \%$ & $6,5 \%$ & $6,5 \%$ & $7,4 \%$ \\
Bindungsstörungen & $11,8 \%$ & $5,1 \%$ & $1,1 \%$ & $14,7 \%$ \\
Regulationsstörungen & $11,8 \%$ & $5,1 \%$ & $42,0 \%$ & $26,5 \%$ \\
Schlafstörungen & $7,1 \%$ & $10,0 \%$ & $2,9 \%$ & $11,8 \%$ \\
Fütter- und Essverhaltensstörungen & $1,2 \%$ & $11,8 \%$ & $4,1 \%$ & $13,2 \%$ \\
Multisystemische Entwicklungsstörungen & $12,9 \%$ & k.A. & $9,5 \%$ & $5,9 \%$ \\
& & & & \\
Achse II & $40,5 / 72,6 \%$ & $52,0 \%$ & $37,2 \%$ & n.u. \\
\hline
\end{tabular}

Besondere Schwierigkeiten gab es in unserer eigenen Untersuchung mit der Klassifikation von Fütterstörungen. Problematisch war die Abgrenzung der Regulationsstörungen, insbesondere vom stimulationssuchend-impulsiven Typ. Sowohl die Kodierung von aggressiven Verhaltensstörungen als auch von affektiven Störungen war schwierig.

Für die Bindungsstörung sind nach wie vor ausführlichere Kriterien unter Berücksichtigung neuere empirischer Daten notwendig. 


\subsubsection{Research Diagnostic Criteria - Preschool Age (RDC-PA)}

Mit dem Auftrag zur Formulierung von Forschungskriterien für die psychiatrische Diagnostik in der frühen Kindheit ist auch eine Arbeitsgruppe von der American Association for Child and Adolescent Psychiatry (AACAP) eingesetzt worden (Task-force 2003).

Im Gegensatz zur DC: 0-3 liegt der Schwerpunkt ausschließlich auf der Adaptation der Kriterien des klinisch-psychiatrischen Syndroms der ersten Achse, hier im DSMIV, unter Verzicht auf die Inauguration eines völlig neuen multiaxialen Systems.

In einem ersten Arbeitsprozess entstanden aus der Sichtung von 17 relevanten DSM-IV-Diagnosen Vorschläge zur Formulierung von Forschungskriterien für eine Adaptation an das Vorschulalter. Es werden sechs neue Untergruppen für Fütterstörungen und zwei für Schlafstörungen vorgeschlagen. Die meisten Kriterien für einzelne Störungen konnten belassen werden, etliche wurden aber inhaltlich modifiziert. Dabei wurden meist die Zeitkriterien oder die Zahl der notwendigen Kriterien reduziert (Tab. 7).

Tabelle 7: Grundsätze der RDC-PA

1. So nah wie möglich an DSM/ICD bleiben

Dies ist u.a vorteilhaft, um für einzelne Störungsbilder Vergleiche zwischen verschiedenen Altersgruppen zu ermöglichen.

2. Herausfragen, nicht hineinfragen

Säuglinge und Kleinkinder können ihre Gedanken und Gefühle nur den kognitiven und verbalen Möglichkeiten ihres Entwicklungsstandes entsprechend äußern. Dieser Umstand darf nicht durch Vermutungen des Untersuchers, kann aber durch die Bewertung eines bestimmten Verhaltens ersetzt werden. Damit können manche Symptome, die berichtet werden müssen, nicht erfasst werden. Sie können allerdings durch die Bewertung eines bestimmten Verhaltens ersetzt werden.

3. Elternverhalten nicht in die Diagnose einbeziehen Im DSM/ICD-Geist bleibt auch die RDC-PA dabei, phänomenologisch und nicht ätiologisch zu klassifizieren.

4. Klare Abgrenzung zu Behinderungen

5. Klare Abgrenzung zu Problemen durch die Untersuchungstechnik

Damit sind Probleme zu Fragen der Bewertung von Intensität und Frequenz von Verhalten und fremdanamnestischer Angaben sowie des Settings gemeint.

Das Altersspektrum ist erweitert auf bis zu 6,11 Jahre (Scheeringa, pers. Mitteilung 2005).

Die RDC-PA sind abrufbar unter http://www.infantinstitute.com/RDC-PA.htm.

Zusammenfassend zeigen sich in allen benutzten Systemen Probleme, die von verschiedenen internationalen Arbeitsgruppen mit Ausrichtung auf ein spezifisches Störungsbild jeweils separat behandelt werden. 


\section{Diskussion}

Durch die Einführung des Klassifikationssystems DC: 0-3R und die Formulierung von Forschungskriterien zur Adaptation klassischer kinder- und jugendpsychiatrischer Störungsbilder an das Kleinkindalter erhält die junge Subspezialität „Säuglings- und Kleinkindpsychiatrie" auch im deutschen Sprachraum einen guten Impuls.

Allerdings bleibt selbst bei modifizierter Anwendung von ICD-10/DSM-IV Kriterien mit Hilfe der RDC-PA auf den entwicklungsbedingt sehr variantenreichen Altersbereich unklar, ob damit physiologische (Durchgangs)phänomene oder „echte“ Störungen bzw. deren Vorläufer beschrieben werden. Dies wird besonders deutlich am Beispiel der Angst- oder Sozialstörungen (Emde 2003).

Die Konzepte der Regulationsstörungen und der Beziehungsklassifikation, die ängstliches und aggressives Verhalten gut erklären können, werden darüber hinaus nicht bearbeitet, dazu verweist die RDC-PA von sich aus auf die DC: 0-3 und deren Experimentalcharakter.

Das Prinzip der Verhaltenskodierung birgt die grundsätzliche Gefahr, dass die diagnostische Qualität des „Leidensdrucks“ und die Rolle der Empathie des Untersuchers als diagnostisch-therapeutischem Werkzeug zu sehr abgedrängt werden. Als Beispiel nennt Emde das Wissen über das Schmerzerleben von Neu- und Frühgeborenen, das uns nur durch die Entschlüsselung von nonverbalen Codes zur Verfügung steht. Es ist inzwischen gut belegt, dass der Gefühlsausdruck bei Kleinkindern in hohem Maße von der Rezeption, der Empathie und den Spiegelprozessen der Bezugsperson abhängt (Fonagy 2000; Stern 1996; Gergely 1996).

Der Ausschluss elterlichen Verhaltens ist damit der schwerwiegendste Kritikpunkt bei der Anwendung der klassischen Systeme und ihrer Adaptationen. Es ist verständlich, dass der Focus auf einem dem Kind innewohnenden Syndrom bleiben soll. Dieses Prinzip steht aber im Widerspruch zu den entwicklungspsychologischen Tatsachen und der klinischen Erfahrung (Emde 2003). Das psychosomatisch organisierte affektive Regulationssystem des Kleinkindes ist untrennbar mit der koregulativen Funktion der Bezugsperson verbunden. Das erklärt die hohe Pervasivität der Störungsmuster und Überschneidungen in den diagnostischen Kategorien. Unserer Ansicht nach weist dies auf die grundlegende, Bedeutung der frühen Bildung von inneren Arbeitsmodellen (Bowlby) als „pluripotente Keimzelle“ der Identitäts- und Persönlichkeitsentwicklung hin.

Objektive Klassifikationsinstrumente sind trotz aller Unzulänglichkeiten dennoch unverzichtbar. Sie unterstützen die Früherkennung und ermöglichen eine rationale Therapieplanung und Evaluation. Sie sind notwendig zur Beurteilung von Längsschnittverläufen und sind nicht zuletzt auch die Grundlage zur Formulierung von Rechtsansprüchen gegenüber Kostenträgern, denn sie erleichtern im Einzelfall die Einordnung in den klinischen oder subklinischen Bereich mit den entsprechenden therapeutischen Konsequenzen (Cantwell 1996, Eppright et al. 1998).

Dennoch sollten psychiatrische Diagnosen bei Säuglingen und Kleinkindern restriktiv vergeben werden.

(c) Vandenhoeck \& Ruprecht GmbH \& Co. KG, Göttingen 
Der notwendige Auf- und Ausbau von spezialisierten Diagnose- und Behandlungszentren für seelische Gesundheit in der frühen Kindheit ist unter den herrschenden Ökonomie- und Qualitätsanforderungen ohne rationale, standardisierte Diagnostik und Klassifikation nicht zu vertreten. Die Klassifikation nach DC: 0-3R ist diesbezüglich eine gutes Instrument. Für die praktische Anwendung innerhalb des in der deutschen Kinder- und Jugendpsychiatrie gut etablierten Multiaxialen Systems (MAS) schlagen wir deshalb zunächst die Nutzung der Achsen I und II und deren Integration vor.

Dies gelingt über die Vergabe der „F98.8“: sonstige näher bezeichnete Störung (hier: mit der DC: 0-3R näher bezeichnete Störung) und den Verweis auf die Achse I der DC: $0-3 R$.

Diagnosen auf der Achse II der DC: 0-3R stellen eine Ergänzung der psychosozialen Belastungen auf der 5. Achse des MAS dar und werden quantitativ mit der PIR-GAS eingeschätzt.

Die Faktoren der Achsen III und IV der DC: 0-3R, („medizinische Diagnose“ und „psychosoziale Belastungsfaktoren“), werden durch das MAS auf der 2. bis 5. Achse besser abgebildet. Die Achse V: „funktionelles Entwicklungsniveau“, die keine Entsprechung im MAS hat, muss klinisch-empirisch noch genauer überprüft werden (Maestro et al. 2002). Deshalb sehen wir von der Integration der Achsen II - V der DC: 0-3R zunächst ab (Tab. 8).

Tabelle 8: Klassifikationsschema für Störungen bei Kindern von 0 bis 5 Jahren

\begin{tabular}{|c|c|}
\hline Allgemeine Form: & Verwendung des Schemas im Arztbrief: \\
\hline Diagnosen nach ICD 10 & Diagnosen nach ICD 10 \\
\hline 1. Achse: Klinisch-psychiatrisches Syndrom & $\begin{array}{l}\text { 1. Achse: Klinisch-psychiatrisches Syndrom (FXX.X) } \\
\text { oder } \\
\text { Sonstige näher bezeichnete Störung (F98.8) }\end{array}$ \\
\hline $\begin{array}{l}\text { 2. Achse: Umschriebene Entwicklungsstö- } \\
\text { rungen }\end{array}$ & $\begin{array}{l}\text { 2. Achse: keine umschriebenen Entwicklungsstörungen } \\
\text { oder } \\
\text { Umschriebene... (Ziffer) }\end{array}$ \\
\hline 3. Achse: Intelligenzniveau & $\begin{array}{l}\text { 3. Achse: Intelligenzniveau (Ziffer oder Klinischer } \\
\text { Eindruck) }\end{array}$ \\
\hline $\begin{array}{l}\text { 4. Achse: Krankheiten aus anderen Kapiteln } \\
\text { nach ICD-10 }\end{array}$ & $\begin{array}{l}\text { 4. Achse: Krankheiten aus anderen Kapiteln nach } \\
\text { ICD-10 (Ziffer) }\end{array}$ \\
\hline 5. Achse: Psychosoziale Belastungsfaktoren & $\begin{array}{l}\text { 5. Achse: Keine psychosozialen Belastungsfaktoren } \\
\text { oder }\end{array}$ \\
\hline 6. Achse: Globales Funktionsniveau & $\begin{array}{l}\text { Psychosoziale Belastungsfaktoren: .... (Ziffer) } \\
\text { 6. Achse: ... soziale Beeinträchtigung (Ziffer) }\end{array}$ \\
\hline Diagnosen nach DC: $0-3 \mathrm{R}$ & Diagnosen nach DC 0-3R: \\
\hline Achse I: Klinisch-psychiatrisches Syndrom & $\begin{array}{l}\text { Achse I: Klinisch-psychiatrisches Syndrom (Ziffer) } \\
\text { oder }\end{array}$ \\
\hline $\begin{array}{l}\text { Achse II: Beziehung zur primären Bezugs- } \\
\text { person }\end{array}$ & $\begin{array}{l}\text { Sonstige näher bezeichnete Störung (800) } \\
\text { Achse II: Beziehung zur primären Bezugsperson... } \\
\text { PIR-GAS }\end{array}$ \\
\hline
\end{tabular}

DC: 0-3R (Diagnostic Classification 0-3, 1. Revision). ZTT, Washington, 2005

(C) Vandenhoeck \& Ruprecht GmbH \& Co. KG, Göttingen 
Die DC: 0-3R kann und sollte auch außerhalb des Fachbereichs Kinder- und Jugendpsychiatrie genutzt werden. Dies betrifft in erster Linie die „Nachbarschaft“ der mit der Problematik vertrauten Sozialpädiatrie (Sozialpädiatrische Zentren, SPZ) aber auch die (beispielsweise in Österreich) in die Pädiatrie integrierte Kinderpsychosomatik und daneben auch den paramedizinischen psychologisch/pädagogischen Versorgungsbereich mit entwicklungsneuropsychologischer Aufgabenstellung und Kompetenz. Voraussetzung ist in jedem Fall eine entsprechende diagnostische Grundkompetenz, spezielle Fort- und Weiterbildung zu seelischer Gesundheit in der frühen Kindheit sowie Einarbeitung in die Handhabung des Systems. Dieser Prozess wird von der „World Association of Infant Mental Health, WAIMH“ (http://waimh.org) und ihrer deutschsprachigen Tochter „Gesellschaft für seelische Gesundheit in der frühen Kindheit, GAIMH e.V.“ (http://www.gaimh.de) unterstützt und gefördert.

Daran angelehnt sollten in den Leitlinien für diejenigen Störungen, die auch im Kleinkindalter relevant sind, spezielle Kriterien für die Altersgruppe der unter 5jährigen aufgenommen werden. Betroffen sind Depression, Angst, Bindungsstörung, Autismus, Ess- und Fütterstörungen, Sozialverhaltensstörungen.

Abschließend sei noch einmal die primäre Einbettung der Thematik innerhalb der kinder- und jugendpsychiatrischen Gemeinschaft verteidigt. Selbstverständlich sollen in der kindlichen Psychodiagnostik die Ressourcen und Stärken im Vordergrund stehen. Die Schwierigkeiten werden ja in der Tat weniger intraindividuell, sondern im Lebens- und Beziehungskontext geortet. Aber gerade deshalb, und weil der Umwelteinfluss auf die Persönlichkeits- und Identitätsentwicklung so prägend ist, muss bei Bedrohung der mentalen Entwicklung kleiner Kinder etwa durch schwerwiegende Fütterstörungen, frühe Bindungsstörungen und offene oder verdeckt-subtile Misshandlungs- und/oder Vernachlässigungssyndrome schon frühzeitig fach-psychotherapeutisch behandelt werden. Jenseits von Beratung und Begleitung bei passageren Störungen ist es die Aufgabe der universitären Kinder- und Jugendpsychiatrie, auch vor dem theoretischen Hintergrund der so genannten Frühstörungskonzepte, durch intensive klinische Forschung die entsprechenden Abgrenzungen zu treffen. Eine rationale Diagnostik, wie sie hier nachgezeichnet wurde, ist der erste Schritt.

\section{Literatur}

Achenbach, T. M.; Rescorla, L. A. (2000): CBCL/1,5-5 \& C-TRF/1,5-5 Profiles. Burlington: University of Vermont, Research Center for Children, Youth, \& Families.

Bayley, N. (1993): Bayley Scales of Infant Development (2nd Edition). Göttingen: Hogrefe.

Bradley, R. H. (1985): The HOME Inventory: Rationale and research. In: J. E. Stevenson (Hrsg.), Recent research in developmental psychopathology (Bd. 4, S. 191-201). Oxford: Pergamon.

Cantwell, D. P. (1996): Classification of child and adolescent psychopathology. J Child Psychol Psychiatry 37: 3-12.

Carter, A. S.; Briggs-Gowan, M. J.; Davis, N. O. (2004): Assessment of young children's socialemotional development and psychopathology: recent advances and recommendations for practice. J Child Psychol Psychiatry, 45(1): 109-134.

(c) Vandenhoeck \& Ruprecht GmbH \& Co. KG, Göttingen 
Cesari, A.; Maestro, S.; Cavallaro, C.; Chilosi, A.; Pecini, C.; Pfanner, L.; Muratori, F. (2003): Diagnostic boundaries between regulatory and multisystem developmental disorders: A clinical study. Infant Ment Health J 24: 365-377.

De Giacomo, A.; Fombonne, E. (1998): Parental recognition of developmental abnormalities in autism. Eur Child Adolesc Psychiatry 7: 131-136.

Drell, M. J.; Siegel, C. H.; Gaensbauer, T. J. (1993): Post-traumatic stress disorder. In C. H. Zeanah (Hrsg.), Handbook of Infant Mental Health. New York: Guilford Press.

Dunitz, M.; Scheer, P. J.; Kvas, E.; Macari, S. (1996): Psychiatric diagnoses in infancy: A comparison. Infant Ment Health J 17: 12-23.

Emde, R. N. (2003): RDC-PA: a major step forward and some issues. J Am Acad Child Adolesc Psychiatry 42: 1513-1516.

Eppright, T. D.; Bradley, S.; Sanfacon, J. A. (1998): The diagnosis of infant psychopathology: current challenges and recent contributions. Child Psychiatry Hum Dev 28: 213-222.

Fonagy, P. (2000): Das Verständnis für geistige Prozesse, die Mutter-Kind-Interaktion und die Entwicklung des Selbst. In: F. Petermann; K. Niebank; H. Scheithauer (Hrsg.), Risiken in der frühkindlichen Entwicklung (S. 241-254). Göttingen: Hogrefe.

Fonagy, P. (2003): The development of psychopathology from infancy to adulthood: The mysterious unfolding of disturbance in time. Infant Ment Health J 24: 212-239.

Frankel, K. A.; Boyum, L. A.; Harmon, R. J. (2004): Diagnoses and presenting symptoms in an infant psychiatry clinic: comparison of two diagnostic systems. J Am Acad Child Adolesc Psychiatry 43: 578-587.

Fox, NA; Kimmerly, NL; Schafer, WD (1991): Attachment to mother/attachment to father: a meta-analysis. Child Dev 62: 210-25.

Gergely, G.; Watson, J. (1996): The social biofeedback theory of parental affect-mirroring: The development of emotional self-awareness and self-control in infancy. International Journal of Psycho-Analysis 77: 1181-1212.

Guedeney, A.; Maestro, S.; (Hrsg.)(2003): Special Issue: The Use of the Diagnostic Classification 0-3. Infant Ment Health J, 24.

Guedeney, N.; Guédeney, A.; Rabouam, C.; Mintz, AS.; Danon, G.; Moralès Huet, M.; Jacquemain, F.; (2003): The Zero-To-Three diagnostic classification: A contribution to the validation of this classification from a sample of 85 under-threes. Infant Ment Health J 24: 313-336.

Keren, M.; Feldman, R.; Tyano, S. (2001): Diagnoses and interactive patterns of infants referred to a community-based infant mental health clinic. J Am Acad Child Adolesc Psychiatry 40: 27-35.

Keren, M.; Feldman, R.; Tyano, S. (2003): A five-year Israeli experience with the DC: 0-3 classification system. Infant Ment Health J 24: 337-348.

Laucht, M.; Esser, G.; Schmidt, M. H. (2000): Längsschnittforschung zur Entwicklungsepidemiologie psychischer Störungen: Zielsetzung, Konzeption und zentrale Befunde der Mannheimer Risikokinderstudie. Zeitschrift für Klinische Psychologie und Psychotherapie 29: 246-262.

Maestro, S.; Muratori, F.; Cavallaro, M. C.; Pei, F.; Stern, D.; Golse, B.; Palacio-Espasa, F. (2002): Attentional skills during the first 6 months of age in autism spectrum disorder. J Am Acad Child Adolesc Psychiatry 41: 1239-1245.

Maldonado-Durán, M.; Helmig, L.; Moody, C.; Fonagy, P.; Fulz, J.; Lartigue, T.; SaucedaGarcia, J. M.; Karacostas, V.; Millhuff, C.; Glinka, J. (2003): The Zero-To-Three diagnostic classification in an infant mental health clinic: Its usefulness and challenges. Infant Ment Health J 24: 378-397.

Papoušek, M. (1995): Kommunikations- und Beziehungsdiagnostik im Säuglingsalter. Zeit- 
schrift für Kinder- und Jugendpsychiatrie 23(Suppl. 1): 18-23.

Papoušek, M.; Schieche, M.; Wurmser, H. (Hrsg.). (2004): Regulationsstörungen der frühen Kindheit. Bern: Huber.

Pavuluri, M. N.; Luk, S. L. (1996): Pattern of preschool behavior problems in New Zealand, using the Behavior Check List. J Paediatr Child Health 32: 132-137.

Remschmidt, H.; Schmidt, M.; Poustka, F. (2001): Multiaxiales Klassifikationssschema für psychische Störungen des Kindes- und Jugendalters nach ICD-10 der WHO. Bern, Göttingen, Toronto, Seattle: Huber.

Resch, F. (1999): Entwicklungspsychopathologie des Kindes- und Jugendalters. Weinheim: Beltz.

Rutter, M.; Sroufe, L. A. (2000): Developmental psychopathology: concepts and challenges. Dev Psychopathol 12: 265-296.

Sameroff, A. J. (1998): Environmental risk factors in infancy. Pediatrics 102(5 Suppl E): 12871292.

Scheeringa, M. S.; Zeanah, C. H.; Drell, M. J.; Larrieu, J. A. (1995): Two approaches to the diagnosis of posttraumatic stress disorder in infancy and early childhood. J Am Acad Child Adolesc Psychiatry 34: 191-200.

Stern, D. N. (1996): Die Lebenserfahrung des Säuglings. Stuttgart: Klett-Cotta.

Stern, D. N. (1998): Die Mutterschaftskonstellation - Eine vergleichende Darstellung verschiedener Formen der Mutter-Kind-Psychotherapie. Stuttgart: Klett-Cotta.

Thomas, A.; Chess, S. (1984): Genesis and evolution of behavioral disorders: from infancy to early adult life. Am J Psychiatry 141: 1-9.

Thomas, J. M.; Benham, A. L.; Gean, M.; Luby, J.; Minde, K.; Turner, S.; Wright, H. H. (1997): Practice parameters for the psychiatric assessment of infants and toddlers (0-36 months). American Academy of Child and Adolescent Psychiatry. J Am Acad Child Adolesc Psychiatry 36(10 Suppl): 21S-36S.

Thomas, J. M.; Clark, R. (1998): Disruptive behavior in the very young child: Diagnostic Classification: 0-3 guides identification of risk factors and relational interventions. Infant Ment Health J 19: 229-244.

Wiefel, A.; Wollenweber, S.; Oepen, G.; Lenz, K.; Lehmkuhl, U.; Biringen, Z. (2005): Emotional availability in infant psychiatry. Infant Ment Health J 26, 392-403.

Zeanah, C. H.; Boris, N. W.; Larrieu, J. A. (1997a): Infant development and developmental risk: a review of the past 10 years. J Am Acad Child Adolesc Psychiatry 36: 165-178.

Zeanah, C. H.; Boris, N. W.; Scheeringa, M. S. (1997b): Psychopathology in infancy. J Child Psychol Psychiatry 38: 81-99.

ZERO-TO-THREE (Hrsg.). (1994): Diagnostic Classification: 0-3. Arlington: National Center for Clinical Infant Programs.

ZERO-TO-THREE (2005): Introducing DC: 0-3R

ZERO-TO-THREE (2005): Diagnostic Classification of Mental Health and Developmental Disorders of Infancy and Early Childhood Revised Edition. Washington: Zero to Three Press.

Korrespondenzadresse: Dr. Andreas Wiefel, Otto-Heubner-Centrum für Kinderund Jugendmedizin der Charité, Sozialpädiatrisches Zentrum für chronisch kranke Kinder und Klinik für Psychiatrie, Psychosomatik und Psychotherapie des Kindesund Jugendalters, Augustenburger Platz 1, 13353 Berlin, fax: 004930450566 923; E-Mail: andreas.wiefel@charite.de 